\title{
Blood pressure reduction and outcome after endovascular therapy with successful reperfusion: a multicenter study
}

\author{
Mohammad Anadani (D) ,' Adam S Arthur, ${ }^{2}$ Ali Alawieh (D) , ${ }^{1}$ Yser Orabi, ${ }^{1}$ \\ Andrei Alexandrov, ${ }^{3}$ Nitin Goyal, ${ }^{3}$ Marios-Nikos Psychogios (D) , ${ }^{4}$ Ilko Maier (D) , \\ Joon-tae Kim, ${ }^{6}$ Saleh G Keyrouz, ${ }^{7}$ Adam de Havenon (D) , ${ }^{8}$ Nils H Petersen, ${ }^{9}$ \\ Abhi Pandhi, ${ }^{10}$ Christa B Swisher, ${ }^{11}$ Ovais Inamullah, ${ }^{11}$ Jan Liman, ${ }^{12}$ Sreeja Kodali, ${ }^{9}$ \\ James A Giles (1) ,7 Michelle Allen, ${ }^{7}$ Stacey Q Wolfe, ${ }^{13}$ Georgios Tsivgoulis, ${ }^{14}$ \\ Bradley A Cagle, ${ }^{13}$ Chesney S Oravec, ${ }^{13}$ Benjamin Gory, ${ }^{15}$ Pierre De Marini, ${ }^{15}$ \\ Peter Kan, ${ }^{16}$ Shareena Rahman, ${ }^{11}$ Sébastien Richard, ${ }^{17}$ Fábio A Nascimento, ${ }^{18}$ \\ Alejandro Spiotta ${ }^{1}$
}

For numbered affiliations see end of article.

\section{Correspondence to} Dr Mohammad Anadani, Medical University of South Carolina, Charleston, SC, USA; manadani@wustl.edu

Dr Alejandro Spiotta; spiotta@ musc.edu

Received 19 October 2019 Revised 19 November 2019 Accepted 20 November 2019 Published Online First

5 December 2019
Check for updates

(C) Author(s) (or their employer(s)) 2020. No commercial re-use. See rights and permissions. Published by BMJ.

To cite: Anadani $\mathrm{M}$,

Arthur AS, Alawieh A, et al.

J Neurolntervent Surg

2020;12:932-936.

\section{ABSTRACT}

Background Elevated systolic blood pressure (SBP) after mechanical thrombectomy (MT) correlates with worse outcome. However, the association between $\mathrm{SBP}$ reduction (SBPr) and outcome after successful reperfusion with MT is not well established.

Objective To investigate the association between SBPr in the first 24 hours after successful reperfusion and the functional and safety outcomes of MT.

Methods A multicenter retrospective study, which included 10 comprehensive stroke centers, was carried out. Patients with acute ischemic stroke and anterior circulation large vessel occlusions who achieved successful reperfusion via MT were included. SBPr was calculated using the formula $100 \times$ ([admission SBP-mean SBP]/admission SBP). Poor outcome was defined as a modified Rankin Scale (mRS) score of 3-6 at 90 days. Safety endpoints included symptomatic intracerebral hemorrhage, mortality, and requirement for hemicraniectomy during admission. A generalized mixed linear model was used to study the association between SBPr and outcomes.

Results A total of 1361 patients were included in the final analysis. SBPr as a continuous variable was inversely associated with poor outcome $(\mathrm{OR}=0.97$; $95 \% \mathrm{Cl} 0.95$ to $0.98 ; p<0.001$ ) but not with the safety outcomes. Subanalysis based on reperfusion status showed that SBPr was associated with lower odds of poor outcome only in patients with complete reperfusion (modified Thrombolysis in Cerebral Infarction $(\mathrm{mTICl} 3))$ but not in patients with incomplete reperfusion ( $\mathrm{mTICl} 2 \mathrm{~b})$. When SBPr was divided into categories $(<1 \%, 1 \%-10 \%, 11 \%-20 \%$, $>20 \%$ ), the rate of poor outcome was highest in the first group.

Conclusion SBPr in the first 24 hours after successful reperfusion was inversely associated with poor outcome. No association between SBPr and safety outcome was found.

\section{INTRODUCTION}

Hypertension is common among patients with stroke and especially those presenting with acute ischemic stroke owing to large vessel occlusions. ${ }^{12}$ Many studies investigating the relation between blood pressure (BP) and outcome after mechanical thrombectomy (MT) have reported a significant association between higher BP and poor outcome, ${ }^{3-7}$ a finding that is consistent with studies from before the thrombectomy era. ${ }^{89}$ However, the relationship between systolic $\mathrm{BP}$ reduction (SBPr) and outcome of MT has not been studied. Previous studies of patients with ischemic stroke suggested a possible detrimental effect of SBP reduction (SBPr) on ischemic stroke outcomes. ${ }^{10-12}$ However, these studies did not include patients who had received MT, and therefore their findings cannot be reliably applied to these patients.

Owing to a lack of randomized trials, there is no consensus on the best approach for BP control after MT, and the last American Heart Association/American Stroke Association (AHA/ASA) guidelines recommended maintaining BP below $180 / 105 \mathrm{~mm} \mathrm{Hg}$ after reperfusion treatment. ${ }^{13}$ However, owing to concern about reperfusion injury and the risk of hemorrhagic complications, SBP reduction to $<140 \mathrm{~mm} \mathrm{Hg}$ after successful reperfusion has become an acceptable practice as demonstrated by a recent survey of the StrokeNet institutions. ${ }^{14}$

Therefore, it is imperative to understand the association between SBPr and the functional and safety outcomes after MT, especially in patients who achieve successful reperfusion with MT. In this study, we investigated the association between SBPr and clinical outcomes after successful reperfusion by means of MT in a large multicenter study. 


\section{METHODS}

\section{PATIENT POPULATION}

The study is an international retrospective multicenter study that includes 10 comprehensive stroke centers across four countries. The study period was between January 2015 and January 2018. Patients were included if they presented with acute ischemic stroke due to anterior circulation large vessel occlusions, and were treated with MT and had successful reperfusion, defined as modified Thrombolysis in Cerebral Infarction (mTICI) score

\begin{tabular}{|c|c|}
\hline Characteristics & Value \\
\hline Age, years, (mean (SD)) & $68(15)$ \\
\hline Female & $677 / 1360(50)$ \\
\hline White & 795/1355 (59) \\
\hline \multicolumn{2}{|l|}{ Medical history } \\
\hline Diabetes & $430 / 1360(32)$ \\
\hline Hypertension & $854 / 1361(63)$ \\
\hline Atrial fibrillation & $500 / 1360(37)$ \\
\hline Hyperlipidemia & 534/1361 (39) \\
\hline \multicolumn{2}{|l|}{ Admission variables } \\
\hline Baseline mRS (median (IQR)) & $0(1)$ \\
\hline Admission NIHSS score, (median (IQR)) & $15(9)$ \\
\hline Onset to Groin (min) (median (IQR)) & $216(174)$ \\
\hline Onset to groin $<6$ hours & 1094/1303 (84) \\
\hline ASPECTS (median (IQR)) & $9(2)$ \\
\hline IV tPA (N (\%)) & $760(55)$ \\
\hline Admission SBP (mean (SD)) & $143(27)$ \\
\hline Mean SBPr (mean (SD)) & $7 \%(16 \%)$ \\
\hline \multicolumn{2}{|l|}{ Etiology } \\
\hline Large artery atherosclerosis & $227 / 1342(17)$ \\
\hline Cardioembolic & $681 / 1342(51)$ \\
\hline Cryptogenic & $365 / 1342(27)$ \\
\hline Other & $69 / 1342(5)$ \\
\hline \multicolumn{2}{|l|}{ Endovascular treatment } \\
\hline \multicolumn{2}{|l|}{ Location of occlusion } \\
\hline ICA & 230/1359 (17) \\
\hline Tandem occlusion & $110 / 1359(8)$ \\
\hline M1 & $763 / 1359(56)$ \\
\hline Distal occlusion & 255/1359 (19) \\
\hline $\mathrm{mTICl} 3$ & $729 / 1360(54)$ \\
\hline Antihypertensive treatment within 24 hours $\dagger$ & $549 / 1009(54)$ \\
\hline \multicolumn{2}{|l|}{ Outcomes } \\
\hline 90-Day mRS 3-6 & $703 / 1361(52)$ \\
\hline 90-Day mortality & 221/1361(16) \\
\hline $\mathrm{slCH}$ & 70/1358 (5) \\
\hline Hemicraniectomy* & $48 / 1136(4)$ \\
\hline
\end{tabular}

*available from $9 / 10$ centers.

†Available from $8 / 10$ centers.

ASPECTS, Alberta Stroke Program Early CT Score; ICA, Internal carotid artery; IV tPA, intravenous tissue plasminogen activator; mRS, modified Rankin Scale; $\mathrm{mTICl}$, modified Thrombolysis in Cerebral Infarction score; NIHSS, National Institutes of Health Stroke Scale; SBP, systolic blood pressure; SBPr, systolic blood pressure reduction; sICH, symptomatic intracerebral hemorrhage. $\geq 2$ b. Additional inclusion criteria were functional independence before the onset of stroke (modified Rankin Scale $(\mathrm{mRS}) \leq 2$ ), Alberta Stroke Program Early CT Score (ASPECTS) $\geq 6$, and the use modern endovascular devices such as stent retriever and/ or direct aspiration. After MT, all patients were admitted to an intensive care unit for postprocedural care. The study was approved by the local institutional review boards, and requirement for consent was waived.

\section{BLOOD PRESSURE MEASUREMENTS}

SBP was recorded on arrival at the emergency room and during the first 24 hours after MT. SBPr was defined according to the following formula: (aSBP $-\mathrm{mSBP} / \mathrm{aSBP}) \times 100$, where aSBP represents admission SBP and $\mathrm{mSBP}$ represents mean SBP during the first 24 hours after MT. ${ }^{15}$ The use of antihypertensive medications was reported by $8 / 10$ centers, but the type and dosing of hypertensive treatment varied between centers and was not available for this study. Blood pressure management and BP measurement protocols were not standardized between centers.

\section{PATIENT AND TREATMENT PARAMETERS}

Baseline variables included age, sex, race, stroke comorbidities (diabetes, hypertension, atrial fibrillation, hyperlipidemia), admission National Institutes of Health Stroke Scale (NIHSS) score, admission ASPECTS, location of the occlusion, intravenous tissue plasminogen activator (IV tPA), and onset-to-groin time. Procedural details included the final mTICI score and procedure time. All patients had final mTICI scores of $\geq 2 \mathrm{~b}$. mTICI and ASPECTS were scored by local operators with no central review.

\section{OUTCOME PARAMETERS}

The primary endpoint of the study was the 90-day functional outcome. Secondary endpoints were symptomatic intracerebral hemorrhage (sICH), 90-day mortality, and requirement for hemicraniectomy during admission (as a surrogate for brain edema). Functional outcome was assessed using the mRS at 90 days. Poor outcome was defined as 90-day mRS score 3-6. sICH was defined as any intraparenchymal, subarachnoid, or intraventricular hemorrhage on postprocedural CT associated with $\geq 4$ points increase in the NIHSS score during the first 72 hours after MT.

\section{STATISTICAL ANALYSIS}

Baseline characteristics were reported as No (\%), mean (SD), or median (IQR). A generalized mixed linear model was used to assess the association between SBPr and clinical outcomes. Two models were constructed. The first model was adjusted for center (as a random variable), age, and admission NIHSS score. The second model was adjusted for center (as a random variable), age, female gender, Caucasian race, diabetes, hypertension, tPA treatment, onset-to-groin time ( $\leq 6$ hours vs $>6$ hours), stroke etiology, ASPECTS, and complete recanalization (mTICI 3 vs mTICI 2b). We attempted to control for admission SBP but that resulted in multicollinearity due to high correlation between SBP and SBPr $(r=0.8 ; \mathrm{P}<0.001)$, therefore, it was excluded from the models. Subanalysis was performed according to use of antihypertensive medications, history of hypertension, admission SBP (>140 mm Hg vs $\leq 140 \mathrm{~mm} \mathrm{Hg}$ ), recanalization status (mTICI 3 vs 2 b), and stroke severity (NIHSS score $<16$ vs $\geq 16$ ). We then divided SBPr into categories (<1\%, 1\%-10\%, 11\%-20\%, and $>20 \%)$. Analysis for association with outcome was repeated including SBPr as categorical variable and using the first category 


\section{Histogram of systolic blood pressure reduction}

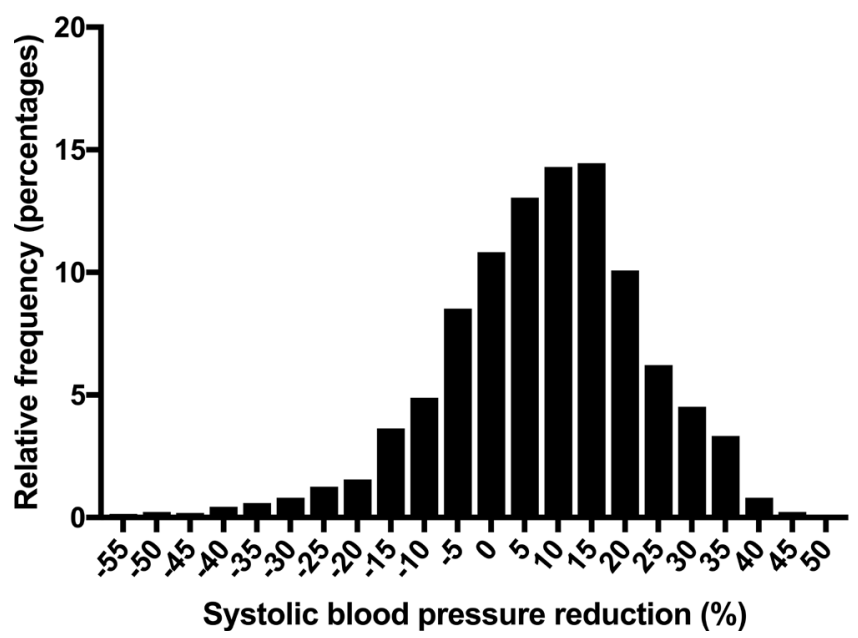

Figure 1 Histogram of systolic blood pressure reduction.

$(<1 \%)$ as a reference. Statistical analyses were performed using SPSS version 25, and figures were created using GraphPad Prism.

\section{RESULTS}

A total of 1454 patients met our inclusion criteria, of whom 1361 had SBP and functional outcome data available and were included in this study. Table 1 summarizes the baseline characteristics of our cohort. Mean (SD) age was 68 (15) years and $50 \%$ of patients were female. Median onset-to-groin time was $216 \mathrm{~min}$ (IQR 174). mTICI 3 was achieved in 54\% of patients. Mean admission SBP (SD) was 143 (27) mm Hg. SBPr distribution is shown in figure 1. A total of 703 (52\%) patients had poor outcome at 90 days after MT. Postprocedural sICH occurred in $5 \%$ and hemicraniectomy was performed in $4 \%$ of patients.

Table 2 depicts the association between SBPr and clinical outcomes. SBPr (as a continuous variable) was inversely associated with poor outcome $(\mathrm{OR}=0.97 ; 95 \% \mathrm{CI} 0.95$ to 0.98 ; $\mathrm{p}<0.001$ ) (table 2). SBPr did not predict sICH, mortality, or hemicraniectomy on multivariable mixed models (table 2).

Results of the subanalysis are reported in table 3. Subanalysis showed the following: (a) SBPr was inversely associated with poor outcome in patients treated with antihypertensive medications in the first 24 hours after MT and also in those who were not treated in this way; (b) there was a trend towards a negative significant association between SBPr and poor outcome only in patients with no history of hypertension but not in patients with a history of hypertension; (c) SBPr was inversely associated with poor outcome in patients with admission SBP $\leq 140 \mathrm{~mm} \mathrm{Hg}$ and
Table 3 Subanalysis based on antihypertensive use in the first 24 hours, history of hypertension, admission systolic blood pressure, and reperfusion status

\begin{tabular}{|c|c|c|c|}
\hline Groups & Model (N) & OR $(95 \% \mathrm{Cl})$ & $P$ value \\
\hline \multirow{2}{*}{$\begin{array}{l}\text { Anti-hypertensive drug } \\
\text { use }\end{array}$} & Model 1 (548) & 0.98 (0.97 to 0.99$)$ & 0.034 \\
\hline & Model 2 (513) & 0.98 (0.97 to 1$)$ & 0.045 \\
\hline \multirow{2}{*}{$\begin{array}{l}\text { No anti-hypertensive } \\
\text { drug use }\end{array}$} & Model 1 (459) & $0.98(0.97$ to 0.99$)$ & 0.014 \\
\hline & Model 2 (420) & 0.98 (0.96 to 0.99$)$ & 0.006 \\
\hline \multirow[t]{2}{*}{ Hypertension } & Model 1 (853) & 0.99 (0.98 to 1.002$)$ & 0.116 \\
\hline & Model 2* (812) & 0.99 (0.98 to 1.003$)$ & 0.156 \\
\hline \multirow[t]{2}{*}{ No hypertension } & Model 1 (506) & 0.98 (0.97 to 1.001$)$ & 0.071 \\
\hline & Model 2* (466) & 0.98 (0.97 to 1.001$)$ & 0.076 \\
\hline \multirow[t]{2}{*}{ Admission SBP >140 } & Model 1 (678) & 0.97 (0.96 to 0.99$)$ & 0.005 \\
\hline & Model 2 (637) & 0.97 (0.95 to 0.99$)$ & 0.008 \\
\hline \multirow[t]{2}{*}{ Admission SBP $\leq 140$} & Model 1 (681) & 0.98 (0.97 to 0.99$)$ & 0.007 \\
\hline & Model 2 (641) & $0.97(0.96$ to 0.99$)$ & 0.001 \\
\hline \multirow[t]{2}{*}{$\mathrm{mTICl} 3$} & Model 1 (728) & 0.98 (0.97 to 0.99$)$ & 0.010 \\
\hline & Model $2^{* *}(691)$ & 0.98 (0.97 to 0.98$)$ & 0.014 \\
\hline \multirow[t]{2}{*}{$\mathrm{mTICl} 2 \mathrm{~b}$} & Model 1 (630) & 0.99 (0.98 to 1.008$)$ & 0.514 \\
\hline & Model $2^{* *}(588)$ & 0.99 (0.98 to 1.007$)$ & 0.406 \\
\hline \multirow[t]{2}{*}{ NIHSS score $<16$} & Model 1 (772) & 0.98 (0.97 to 0.99$)$ & 0.002 \\
\hline & Model $2^{* *}(682)$ & 0.98 (0.97 to 0.99$)$ & 0.005 \\
\hline \multirow[t]{2}{*}{ NIHSS score $\geq 16$} & Model 1 (627) & 1.001 (0.98 to 1.01$)$ & 0.89 \\
\hline & Model 2** (589) & 1.001 (0.98 to 1.01$)$ & 0.86 \\
\hline
\end{tabular}

Model 1 adjusted for center, age, admission NIHSS.

Model 2 adjusted for age, female, Caucasian race, admission ASPECTS, diabetes, hypertension, atrial fibrillation, admission NIHSS, IV tPA, onset-to-groin time, etiology, and complete recanalization ( $\mathrm{mTICI} 3$ ).

ASPECTS, Alberta Stroke Program Early CT Score; $\mathrm{mTICl}$, modified Thrombolysis in Cerebral Infarction ; NIHSS, National Institutes of Health Stroke Scale; SBP, systolic blood pressure; tPA, tissue plasminogen activator.

also in those with admission SBP $>140 \mathrm{~mm} \mathrm{Hg}$; (d) SBPr was inversely associated with poor outcome only in patients with mTICI 3 but not in those with mTICI 2b; (e) SBPr was inversely associated with poor outcome in patients with mild-moderate stroke (NIHSS score $<16$ ) but not in those with severe stroke (NIHSS score $\geq 16$ ).

When SBPr was included as categorical variable $(<1 \%$ (reference), 1\%-10\%, 11\%-20\%,>20\%), all categories were associated with lower odds of poor outcome. However, the association was significant only for the $1 \%-10 \%$ category $(\mathrm{OR}=0.7 ; 95 \% \mathrm{CI}$ 0.45 to 0.99$)$ but not for the $11 \%-20 \%(\mathrm{OR}=0.76 ; 95 \% \mathrm{CI}$

Table 2 Association between systolic blood pressure reduction and clinical outcomes

\begin{tabular}{|c|c|c|c|c|c|c|}
\hline \multirow[b]{2}{*}{ Outcomes } & \multicolumn{3}{|c|}{ Model 1} & \multicolumn{3}{|c|}{ Model 2} \\
\hline & $\mathrm{N}$ & OR $(95 \% \mathrm{Cl})$ & $P$ value & $\mathrm{N}$ & OR $(95 \% \mathrm{Cl})$ & $P$ value \\
\hline Poor outcome & 1359 & 0.99 (0.98 to 0.99$)$ & 0.007 & 1278 & 0.97 (0.95 to 0.98$)$ & $<0.001$ \\
\hline Mortality & 1359 & 0.99 (0.98 to 1$)$ & 0.265 & 1278 & 0.99 (0.97 to 1.007$)$ & 0.602 \\
\hline sICH & 1356 & $1(0.98$ to 1.01$)$ & 0.08 & 1275 & $1(0.98$ to 1.01$)$ & 0.846 \\
\hline Hemicraniectomy & 1356 & 0.99 (0.97 to 1.01$)$ & 0.642 & 1142 & 0.99 (0.97 to 1.01$)$ & 0.88 \\
\hline
\end{tabular}

Model 1 adjusted for center, age, admission NIHSS.

Model 2 adjusted for age, female, Caucasian race, admission ASPECTS, diabetes, hypertension, atrial fibrillation, admission NIHSS, IV tPA, onset-to-groin time, etiology, and complete recanalization (mTICI3).

ASPECTS, Alberta Stroke Program Early CT Score; mTICI, modified Thrombolysis in Cerebal Infarction ; NIHSS, National Institutes of Health Stroke Scale; sICH, symptomatic intracerebral hemorrhage; tPA, tissue plasminogen activator. 


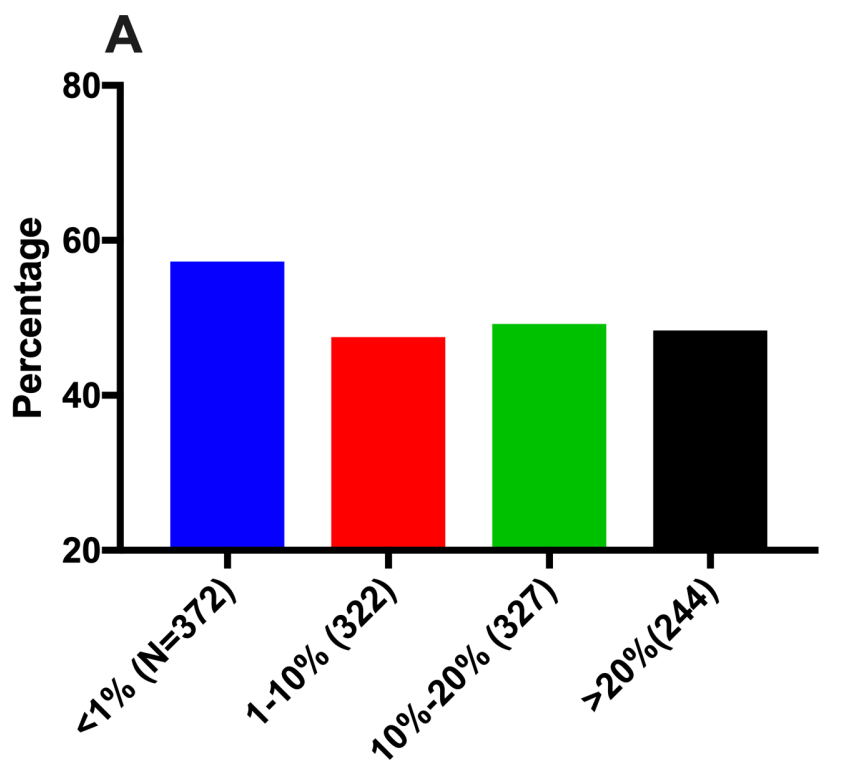

B

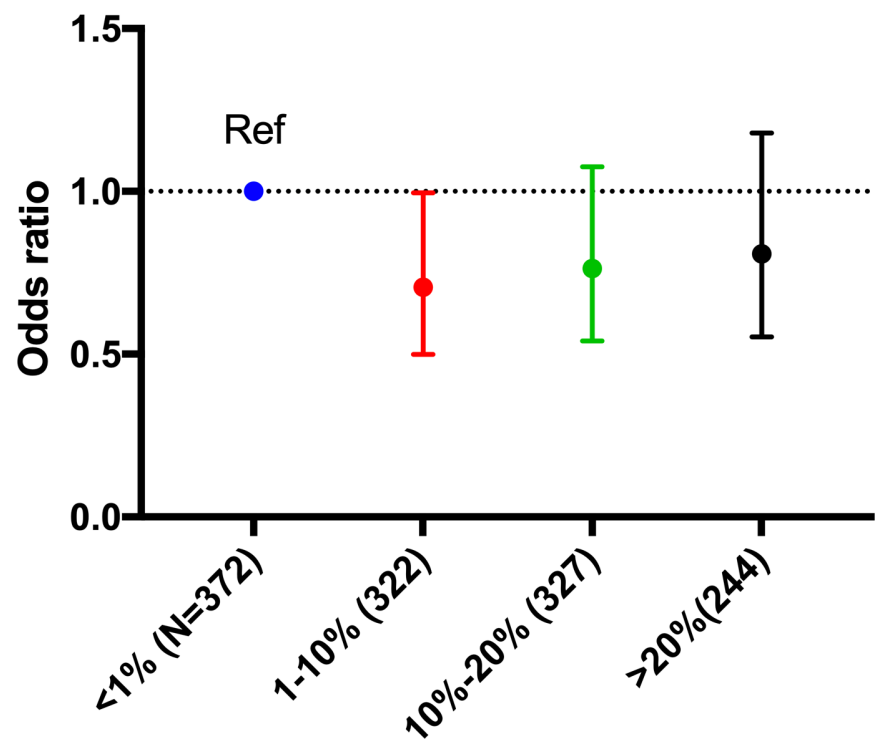

Figure 2 (A) Distribution of poor outcome across 4 SBPr categories $(<1 \%, 1 \%-10 \%, 11 \%-20 \%$, and $>20 \%)$; (B) OR and $95 \% \mathrm{Cl}$ of the association between SBPr categories and poor outcome $(<1 \%$ category was used as a reference). SBPr, systolic blood pressure reduction.

0.54 to 1.07$)$ or $>20 \%$ categories $(\mathrm{OR}=0.8 ; 95 \% \mathrm{CI} 0.55$ to 1.18 ; figure 2).

\section{DISCUSSION}

Blood pressure management is a key challenge in acute ischemic stroke treatment, especially in patients who achieve successful reperfusion with endovascular therapy. Cumulative evidence supports a link between higher BP on admission or after MT and worse functional outcome. ${ }^{3-7}$ However, the effect of SBPr in the first 24 hours on outcome after successful reperfusion is not well understood. Literature from before the MT era provided conflicting results. In a study of 115 patients with ischemic stroke presenting within 24 hours of symptoms onset, higher SBPr was observed in the group with poor outcome $(m R S>2)$ than in the group with a good outcome. ${ }^{11}$ The association between SBPr and worse outcomes was independent of antihypertensive treatment, and the authors concluded that even spontaneous SBP reduction after acute ischemic stroke could be harmful. In another study of 304 patients, a drop in SBP of $>20 \mathrm{~mm} \mathrm{Hg}$ was associated with an early neurological deterioration and poor long-term functional outcome. ${ }^{10}$ In addition, a post hoc analysis of the Intravenous Nimodipine West European Stroke Trial (INWEST) trial demonstrated an association between diastolic BP reduction (but not SBP reduction) after acute stroke and worse outcome. ${ }^{15}$ In contrast, SBPr was found to correlate with an early neurological improvement after intravenous thrombolysis. ${ }^{16}$ The above-mentioned studies did not take into account reperfusion status or the causes of stroke, which could explain some of the inconsistencies.

In our study, the focus was on patients who achieved successful reperfusion, as this subgroup of patients is the most likely to benefit from BP reduction to reduce reperfusion injury. ${ }^{2}$ A weak negative association between SBPr and poor outcome after successful reperfusion was identified. Our study differs from previous studies in many aspects. First, we included a homogeneous group of patients with a relatively similar recanalization status and location of occlusion (anterior circulation). Second, unlike most of the previous studies, we were able to account for admission ASPECTS as a surrogate for initial infarct volume. Third, our study was significantly larger than some of the abovementioned studies, allowing for robust statistical analysis. These differences could explain, at least in part, the inconsistencies between our results and those of previous studies.

Several factors may modify the effect of BP on outcome after MT, including reperfusion status, history of hypertension, and cause of the stroke. In our study, SBPr was associated with a lower odds of poor outcome only in patients with complete reperfusion, but not in those with incomplete reperfusion. This finding is not surprising as patients with incomplete reperfusion may require higher SBP to support retrograde collateral supply to the territory that remains occluded in an antegrade manner. Therefore, SBP reduction in this subgroup of patients may not be beneficial and could be detrimental.

When patients were divided according to their history of hypertension, a trend toward significant association between SBPr and poor outcome was seen in patients without a known history of hypertension, but not in those with no history of hypertension. This finding is probably related to the fact that the autoregulation curve is shifted to the right in patients with a history of hypertension. ${ }^{2}$ Therefore, patients with a history of hypertension can tolerate higher SBP and may not benefit from lowering SBP after MT. This finding, however, has to be interpreted with caution because a history of hypertension in our cohort was reported by patient or family on admission, and thus it is likely that patients with undiagnosed hypertension were included in the (no hypertension) group.

Furthermore, it is known that SBP drops spontaneously in the first few days after an acute stroke and especially in a subgroup of patients who achieve successful reperfusion with intravenous or intra-arterial therapy. ${ }^{2}$ Therefore, it was not clear if the association between SBPr and outcome identified in the main analysis, was limited to spontaneous SBPr. In an attempt to investigate this finding further, we divided patients according to antihypertensive use in the first 24 hours. We found that SBPr was associated with a lower odds of poor outcome regardless of antihypertensive use in the first 24 hours.

We divided SBPr into categories ( $<1 \%$ (reference), 1\%-10\%, $11 \%-20 \%,>20 \%)$ to understand the shape of the relationship 
between SBPr and outcome. Poor outcome was more common in the first group, a finding that is probably due to a significant increase in SBP in the first 24 hours in the first group (mean SBPr $-11 \%)$. The odds of poor outcome were lower in the other three groups $(1 \%-10 \%, 11 \%-20 \%,>20 \%)$ than in the first group $(<1 \%)$. Therefore, it is possible that preventing an increase in SBP is more important than reducing SBP in the first 24 hours after successful recanalization.

Our study has a number of limitations that are mainly related to its retrospective, non-randomized design. First, SBPr was calculated based on a summary of BP readings on admission and during the 24 hours after reperfusion. Therefore, it is still unclear if the timing of BP reduction modifies the association between SBPr and outcome. It is possible that rapid reduction of SBP can be detrimental, especially that cerebral blood flow can be impaired even after complete reperfusion, the so called 'no-reflow phenomenon'. ${ }^{17}$ Second, the type and doses of the antihypertensive medications were not available for this study; therefore, we limited the analysis to whether or not antihypertensive medications were used. Third, owing to heterogeneous protocols after MT imaging, we could not obtain the infarct volume to assess the relation between SBPr and infarct extension. Fourth, our study included only patients with ASPECTS $\geq 6$ and anterior circulation strokes; therefore, our findings may not be applicable to patients with larger initial infarct volume or posterior circulation strokes.

\section{CONCLUSION}

SBPr in the first 24 hoursafter successful reperfusion was inversely associated with poor outcome. The association between blood pressure reduction and functional outcome seems to be more significant in patients with complete reperfusion and without a history of hypertension.

\section{Author affiliations}

'Neurosurgery, Medical University of South Carolina, Charleston, South Carolina, USA

${ }^{2}$ Neurosurgery, University of Tennessee Health Science Center, Memphis, Memphis, USA

${ }^{3}$ Neurology, University of Tennessee Health Science Center, Memphis, Tennessee, USA

${ }^{4}$ Neuroradiology, University Medicine Goettingen, Goettingen, NS, Germany

${ }^{5}$ Neurology, University Medicine Goettingen, Goettingen, NS, Germany

${ }^{6}$ Chonnam, Korea (the Democratic People's Republic of)

${ }^{7}$ Washington University School of Medicine in Saint Louis, Saint Louis, Missouri, USA

${ }^{8}$ Department of Neurology, University of Utah, Salt Lake City, Utah, USA

${ }^{9}$ Yale Univ, New Heaven, Connecticut, USA

${ }^{10}$ Neurology, University of Tennessee Health Science Center College of Medicine,

Memphis, Tennessee, USA

${ }^{11}$ Duke University Hospital, Durham, North Carolina, USA

${ }^{12}$ Department of Neurology, Universitatsklinikum Gottingen, Gottingen, Niedersachsen, Germany

${ }^{13}$ Neurosurgery, Wake Forest School of Medicine, Winston Salem, North Carolina, USA

${ }^{14}$ Second Department of Neurology, "Attikon" Hospital, School of Medicine,

University of Athens, Athens, Greece

${ }^{15}$ Department of Diagnostic and Interventional Neuroradiology, CHRU Nancy, Nancy, Lorraine, France

${ }^{16}$ Department of Neurosurgery, Baylor College of Medicine, Houston, Texas, USA

${ }^{17}$ Neurology Stroke Unit, University Hospital Centre Nancy, Nancy, France

${ }^{18}$ Department of Neurology, Baylor College of Medicine, Houston, Texas, USA

Twitter Abhi Pandhi @abhipandhi and James A Giles@neurogiles

Contributors All authors have: provided a substantial contribution to the conception and design of the studies and/or the acquisition and/or the analysis of the data and/or the interpretation of the data. They have drafted the work or revised it for significant intellectual content and approved the final version of the manuscript. They agree to be accountable for all aspects of the work, including its accuracy and integrity.

Funding The authors have not declared a specific grant for this research from any funding agency in the public, commercial or not-for-profit sectors.

Competing interests AS has honoris for consulting for Penumbra and Cerenovus. M-NP received honoraria; modest; received speakers' honoraria from Siemens Healthineers.SQW reports U24NS107235- 01 NIH/NINDS StrokeNET Site Co-I. The other authors report no conflicts.

Patient consent for publication Not required.

Provenance and peer review Not commissioned; externally peer reviewed.

Data availability statement Data are available upon reasonable request.

\section{ORCID iDs}

Mohammad Anadani http://orcid.org/0000-0002-7813-2949

Ali Alawieh http://orcid.org/0000-0003-2601-8850

Marios-Nikos Psychogios http://orcid.org/0000-0002-0016-414X

Ilko Maier http://orcid.org/0000-0001-6988-8878

Adam de Havenon http://orcid.org/0000-0001-8178-8597

James A Giles http://orcid.org/0000-0002-3197-3062

\section{REFERENCES}

1 Qureshi Al. Acute hypertensive response in patients with stroke: pathophysiology and management. Circulation 2008;118:176-87.

2 Regenhardt RW, Das AS, Stapleton CJ, et al. Blood pressure and penumbral sustenance in stroke from large vessel occlusion. Front Neurol 2017;8:317.

3 Anadani M, Orabi Y, Alawieh A, et al. Blood pressure and outcome post mechanical thrombectomy. J Clin Neurosci 2019;62:94-9.

4 Anadani M, Orabi MY, Alawieh A, et al. Blood pressure and outcome after mechanical thrombectomy with successful revascularization. Stroke 2019;50:2448-54.

5 Goyal N, Tsivgoulis G, Iftikhar S, et al. Admission systolic blood pressure and outcomes in large vessel occlusion strokes treated with endovascular treatment. J Neurointerv Surg 2017:9:451-4.

6 Goyal N, Tsivgoulis G, Pandhi A, et al. Blood pressure levels post mechanical thrombectomy and outcomes in large vessel occlusion strokes. Neurology 2017;89:540-7.

7 Mistry E, Mehta T, Mistry A, et al. Blood pressure variability and neurologic outcome after endovascular thrombectomy: secondary analysis of the best study. Stroke Aha 2019.

8 Ahmed N, Wahlgren N, Brainin M, et al. Relationship of blood pressure, antihypertensive therapy, and outcome in ischemic stroke treated with intravenous thrombolysis: retrospective analysis from safe implementation of thrombolysis in Stroke-International stroke thrombolysis register (SITS-ISTR). Stroke 2009;40:2442-9.

9 Ahmed N, Wahlgren G. High initial blood pressure after acute stroke is associated with poor functional outcome. J Intern Med 2001;249:467-73.

10 Castillo J, Leira R, García MM, et al. Blood pressure decrease during the acute phase of ischemic stroke is associated with brain injury and poor stroke outcome. Stroke 2004;35:520-6.

11 Oliveira-Filho J, Silva SCS, Trabuco CC, et al. Detrimental effect of blood pressure reduction in the first 24 hours of acute stroke onset. Neurology 2003;61:1047-51.

12 Lisk DR, Grotta JC, Lamki LM, et al. Should hypertension be treated after acute stroke? A randomized controlled trial using single photon emission computed tomography. Arch Neurol 1993;50:855-62.

13 Powers WJ, Rabinstein AA, Ackerson T, et al. 2018 guidelines for the early management of patients with acute ischemic stroke: a guideline for healthcare professionals from the American heart Association/American stroke association. Stroke 2018;49:e46-110.

14 Mistry EA, Mayer SA, Khatri P. Blood pressure management after mechanical thrombectomy for acute ischemic stroke: a survey of the strokenet sites. J Stroke Cerebrovasc Dis 2018;27:2474-8.

15 Ahmed N, Näsman P, Wahlgren NG. Effect of intravenous nimodipine on blood pressure and outcome after acute stroke. Stroke 2000;31:1250-5.

16 Gill D, Cox T, Aravind A, et al. A fall in systolic blood pressure 24 hours after thrombolysis for acute ischemic stroke is associated with early neurological recovery. $J$ Stroke Cerebrovasc Dis 2016;25:1539-43.

17 Susanne Wegener. Improving cerebral blood flow after arterial recanalization: a novel therapeutic strategy in stroke. Int J Mol Sci 2017;18:2669. 